\title{
FISICA E REALISMO
}

\author{
NINO ZANGHİ (*)
}

SunTO. - La questione del realismo in fisica è affrontata seguendo la strada tracciata da Einstein e Bell. Dopo aver esposto il problema della completezza della meccanica quantistica e discusso alcuni aspetti del dibattito tra Einstein e Bohr, si indicano quali debbano essere i requisiti di una formulazione della meccanica quantistica che non sia basata su nozioni vaghe e imprecise come "misura" o "osservatore". Si mostra infine come una teoria fisica che descrive "roba" nello spazio che evolve nel corso del tempo renda trasparente il legame tra teoria e realtà empirica. La conclusione è di sapore kantiano.

ABSTRACT. - The question of realism in physics is addressed by following the path laid out by Einstein and Bell. After an exposition of the problem of the completeness of quantum mechanics and a discussion of some aspects of the debate between Einstein and Bohr, we outline what are the requirements of a formulation of quantum mechanics that is not based on such vague and imprecise notions as " measurement" or " observer." Finally, we show that a physical theory that describes "stuff" in the space that evolves over time makes transparent the relationship between theory and empirical reality. The conclusion has a Kantian flavor.

\section{LA DOTTRINA DI COPENAGHEN}

Negli anni venti del XX secolo, il fisico danese Niels Bohr, a quel tempo probabilmente il fisico quantistico più autorevole ed influente, incominciò a difendere l'idea che l'atteggiamento realista degli scienziati fosse infantile e non scientifico e propose quella che ancora oggi è chiamata l'interpretazione di Copenaghen della meccanica quantistica. Secondo il punto di vista di Copenaghen, la realtà è divisa in due

(*) Dipartimento di Fisica dell'Università di Genova, Italia.

E-mail: zanghi@ge.infn.it 
mondi, il microscopico e il macroscopico, il classico e il quantistico, il mondo regolato dalla logica classica e quello regolato dalla logica quantistica. Sebbene non sia chiaro dove stia il confine tra questi due mondi, e come questa dualità sia compatibile con il fatto che le mele e le sedie consistano d'elettroni e d'altre particelle, la dottrina di Copenaghen è diventata l'ortodossia, vale a dire, è diventata non solo il punto di vista maggioritario tra i fisici, ma anche il dogma. Nonostante l'omaggio di prammatica al dogma, questa divisione arbitraria del mondo e il ruolo peculiare dell'osservatore nella formulazione della teoria hanno da sempre lasciato perplessi molti fisici.

Richard Feynman, uno dei padri della moderna elettrodinamica quantistica, espresse così la sua perplessità:

Tutto ciò mi rende estremamente confuso, specialmente quando consideriamo che anche se possiamo consistentemente considerarci osservatori esterni quando guardiamo il resto del mondo, il resto del mondo allo stesso tempo ci sta osservando ... Vuol forse dire che le mie osservazioni diventano reali solo quando osservo un osservatore che osserva qualcosa che accade? Questo è veramente un punto di vista orribile. Ritenete seriamente che senza un osservatore non ci sia una realtà? Quale osservatore? Una mosca è un osservatore? Una stella è un osservatore? C'era qualche realtà antecedente all'anno 109 prima di Cristo, prima che si sviluppasse la vita? Oppure site voi l'osservatore? $\mathrm{Ci}$ sarà qualche realtà per il mondo dopo che sarete morti? Conosco un certo numero di fisici rispettabili che ha stipulato un'assicurazione sulla vita (Feynman, 2002).

Albert Einstein fu tra i pochi che reagirono con vigore alla dottrina di Copenaghen. Nel volume in suo onore, curato da P. A. Schlipp e pubblicato nel 1949, Einstein tracciava un bilancio del suo contributo alla fisica del XX secolo e delle ragioni che lo avevano portato al rifiuto della dottrina di Copenaghen.

Da un lato, Einstein non esitava a riconoscere l'importante e duraturo contributo della meccanica quantistica allo sviluppo della fisica:

[...] io riconosco pienamente l'importantissimo progresso che la teoria statistica dei quanti ha fatto compiere alla fisica teorica. Nel campo dei problemi meccanici — cioè dovunque è possibile considerare l'interazione delle strutture e delle loro parti con sufficiente precisione, postulando un'energia potenziale fra i punti materiali — questa teoria costituisce ancora oggi un sistema capace, pur nel suo carattere ristret- 
to, di descrivere correttamente le relazioni empiriche fra fenomeni specificabili, in modo corrispondente alle previsioni della teoria: essa è finora la sola che unisca il duplice carattere corpuscolare e ondulatorio della materia in modo logicamente soddisfacente: e le relazioni in essa contenute sono, entro i limiti naturali fissati dalla relazione d'indeterminazione, complete. Le relazioni formali contenute in questa teoria cioè il suo intero formalismo matematico - dovranno essere contenute probabilmente, sotto forma di deduzioni logiche, in ogni teoria non inutile del futuro (Einstein, 1979).

D'altro canto non esitava ad evidenziare le difficoltà della teoria quantistica:

Ciò che non mi soddisfa in questa teoria, in linea di principio, è il suo atteggiamento verso ciò che mi sembra essere lo scopo programmatico della fisica stessa: la descrizione completa di ogni situazione reale (individuale) che si suppone possa esistere indipendentemente da ogni atto di osservazione o di verifica (ibid.).

E indicava una possibile strada per lo sviluppo della teoria:

[...] la teoria statistica dei quanti - nel caso che gli sforzi compiuti per ottenere una descrizione fisica completa abbiano avuto successo verrebbe a prendere, nel quadro della fisica futura, un posto approssimativamente analogo a quello della meccanica statistica nei quadro della meccanica classica. Io sono fermamente convinto che lo sviluppo della fisica teorica sarà di questo tipo; ma il cammino sarà lungo e difficile (ibid.).

\section{EINSTEIN E IL PROBLEMA DELLA COMPLETEZZA}

Incominciamo col chiarire che cosa il problema non è. L'adeguatezza empirica di una teoria fisica riguarda un ben circostanziato dominio di validità che non sempre copre la vasta gamma di fenomeni che si osservano in natura; in questo senso, qualunque teoria fisica sarebbe da ritenersi incompleta. Inoltre, la nostra conoscenza empirica del mondo muta nel corso degli anni: la meccanica celeste newtoniana ha ceduto il passo alla moderna meccanica celeste einsteniana che la contiene come caso limite e la termodinamica è stata assorbita dalla moderna meccanica statistica. La "teoria del tutto" di oggi, se soprav- 
vivrà al vaglio dell'esperienza - e alla critica roditrice dei topi - probabilmente diventerà una delle teorie approssimate di domani. In questo senso, nessuna teoria scientifica, per sua natura, fornisce una descrizione completa della realtà. Se, come scriveva Novalis, le teorie sono come reti da pesca che si gettano nel mare, per quanto fini siano le maglie, ci saranno sempre dei pesci che sfuggiranno. Ma questo non è il senso d'incompletezza che Einstein aveva in mente.

Il problema della completezza posto da Einstein riguarda piuttosto la natura della relazione tra il quadro concettuale della teoria espresso da una ben definita struttura matematica, usualmente organizzata in un sistema assiomatico - e il contenuto empirico della teoria nel dominio di validità della teoria stessa. Se il lettore trova questa affermazione un po' oscura (e probabilmente lo è), lo preghiamo di pazientare e di seguirci nel percorso che segue.

Nel famoso articolo pubblicato nel 1935 in collaborazione con Boris Podolsky e Nathan Rosen, Einstein intendeva dare una dimostrazione certa che la funzione d'onda non fornisce una rappresentazione completa della realtà fisica; si tratta del famoso "argomento EPR". Tuttavia, Einstein non fu mai soddisfatto dello stile di quest'articolo. Nella sua Autobiografia Scientifica, Einstein ripresenta in altra forma l'argomento EPR: il ragionamento è ineccepibile e si basa su una sola premessa, il principio di località. Senza entrare nel merito di questo principio, ci basti qui ricordare che per decenni è stato considerato uno dei pilastri della fisica, uno dei principi più certi su cui basare la nostra conoscenza della natura. Per una messa in discussione di questo principio bisogna arrivare al 1964, l'anno in cui il fisico irlandese John Bell pubblicò la dimostrazione dell'incompatibilità di questo principio con la meccanica quantistica.

A supporto della tesi d'incompletezza della meccanica quantistica, Einstein utilizza anche l'argomentazione a cui era pervenuto Schrödinger in maniera del tutto indipendente. Questa argomentazione era stata presentata da Schrödinger all'interno di un articolo d'ampio respiro sullo stato della meccanica quantistica che aveva pubblicato nello stesso anno dell'articolo EPR. Si tratta del famoso articolo "Die gegenwartige Situation in der Quantenmechanik", colloquialmente noto come l'articolo del "gatto".

Einstein considera un atomo radioattivo con un tempo di decadimento medio ben definito, localizzato in modo praticamente esatto in un punto dello spazio. Nel formalismo matematico della meccanica 
quantistica, il processo radioattivo d'emissione di una particella leggera è descritto da una funzione in tre dimensioni (la funzione d'onda della particella leggera) che nell'istante iniziale è diversa da zero solo all'interno della regione occupata dall'atomo, ma che, negli istanti di tempo successivi, si espande nello spazio esterno. Questa funzione fornisce la probabilità che la particella, in un certo istante arbitrario, si trovi in una data parte dello spazio, ma non implica alcun'affermazione sul momento della disintegrazione dell'atomo radioattivo.

Alla domanda se possiamo ritenere questa descrizione teorica come la descrizione completa della disintegrazione del singolo atomo, Einstein risponde negativamente:

Immediatamente la risposta più attendibile è: No. Infatti, si è portati immediatamente a pensare che il singolo atomo decada in un tempo ben definito; ma la descrizione compiuta per mezzo della funzione [d'onda] non implica affatto un valore temporale ben definito. Quindi, se il singolo atomo ha un tempo di disintegrazione definito, allora nei riguardi del singolo atomo la descrizione compiuta per mezzo della funzione [d'onda] dev'essere interpretata come una descrizione incompleta. In questo caso, la funzione [d'onda] dev'essere considerata come la descrizione, non di un singolo sistema, ma di un insieme ideale di sistemi. Si è portati cosi alla convinzione che una descrizione completa di un singolo sistema, alla fin fine, debba essere possibile; ma per una descrizione completa di questo genere non c'è posto nel sistema concettuale della teoria statistica dei quanti (ibid.).

Dopo di che anticipa una possibile obiezione al suo ragionamento:

Questo ragionamento sta in piedi o cade se sta in piedi o cade l'affermazione che esista effettivamente qualcosa di simile a un tempo definito di disintegrazione del singolo atomo (un istante di tempo che esista indipendentemente da ogni osservazione); ma quest'affermazione, dal mio punto di vista, non è soltanto arbitraria, ma addirittura priva di senso. Affermare l'esistenza di un istante preciso in cui avviene la disintegrazione avrebbe senso solo se potessi determinare empiricamente, in linea di principio, quest'istante. Ma questa determinazione (che, in ultima analisi, porta al tentativo di dimostrare l'esistenza della particella al di là della barriera di potenziale) implicherebbe una perturbazione ben definita del sistema di cui ci stiamo occupando, cosicché il risultato di essa non permetterebbe di trarre alcuna conclusione sullo stato del sistema non perturbato. Quindi, la supposizione che un atomo radioattivo abbia un tempo di disintegrazione ben definito non 
è giustificata assolutamente da nulla; e quindi non è dimostrato nemmeno che la funzione d'onda non possa essere concepita come una descrizione completa del singolo sistema. Tutta questa pretesa difficoltà deriva dal fatto che si postula come "reale" una cosa che non è osservabile. (Questa sarebbe la risposta del teorico quantistico.) (ibid.)

Nella risposta del teorico quantistico, così ben tratteggiata da Einstein, si scorge quell'atteggiamento anti-metafisico che è stato molto salutare per lo sviluppo della scienza moderna. Man mano che sondiamo il mondo nelle regioni remote dall'esperienza ordinaria non abbiamo alcun diritto di aspettarci che le nozioni familiari siano adeguate; siamo già molto fortunati ad avere regole di calcolo e procedure sperimentali che funzionano. In altre parole, non dovremmo stupirci se nel mondo microscopico si offuscano le categorie tradizionali di pensiero, come l'idea che esista un tempo ben definito per l'accadimento di un evento.

Tuttavia, proprio riflettendo su questo aspetto del problema - il rapporto tra quadro concettuale e realtà - Einstein arrivò ad un capovolgimento del ragionamento del teorico quantistico. Al contrario, Bohr e i cultori della dottrina di Copenaghen, arrivarono alla convinzione opposta: ritennero che era impossibile andare oltre le categorie di quel quadro concettuale "classico" forgiato dal mondo dell'esperienza quotidiana. Alla risposta del teorico quantistico Einstein muove dapprima un'obiezione filosofica, poi enuncia un criterio metodologico molto generale e infine passa all'argomentazione di Schrödinger del 1935.

\section{EINSTEIN E LA QUESTIONE DEL QUADRO CONCETTUALE}

L'obiezione filosofica di Einstein riguarda l'atteggiamento positivista fondamentale che è alla base del ragionamento del teorico quantistico, un atteggiamento che, secondo Einstein, finisce per confondersi con il principio del vescovo George Berkeley che "essere" è "essere percepito". Per Einstein, al contrario, la giustificazione di un costrutto teorico non è nella sua derivazione dai dati dei sensi. Secondo Einstein, una tale derivazione non è mai possibile, nemmeno nell'ambito del pensiero pre-scientifico: "la giustificazione dei costrutti teorici, che per noi rappresentano la realtà, sta soltanto nella loro capacità di rendere intelligibile ciò che è dato dai sensi" (ibid.) 
Einstein propone quindi il seguente criterio metodologico:

Non ci si può chiedere soltanto se esista un istante di tempo ben definito della trasformazione di un singolo atomo, ma occorre chiedersi piuttosto: "È ragionevole, nel quadro della nostra costruzione teorica complessiva, supporre l'esistenza di un punto temporale ben definito della trasformazione di un singolo atomo?" Non ci si può neppure chiedere che cosa significhi quest'affermazione. $\mathrm{Ci}$ si può chiedere soltanto se una proposizione del genere, nel quadro del sistema concettuale scelto, e tenendo presente la sua capacità di afferrare teoricamente ciò che è dato empiricamente, sia ragionevole o no (ibid.).

Se accettiamo questo criterio, ci troviamo di fronte a due opzioni. La prima consiste nell'assumere un quadro concettuale in cui la funzione d'onda non fornisce una rappresentazione del singolo sistema individuale, bensì di un insieme di sistemi identicamente preparati (che hanno in comune l'essere associati alla stessa funzione d'onda); in tal caso possiamo tranquillamente ammettere che esista un istante di tempo definito in cui il singolo atomo radioattivo decade. La seconda opzione è assumere un quadro concettuale in cui la funzione d'onda è considerata come la descrizione completa del singolo sistema; in tal caso siamo legittimati a rifiutare che esista uno specifico tempo di decadimento del singolo atomo radioattivo.

Per Einstein la seconda opzione deve essere scartata sulla base dell'argomentazione fornita da Schrödinger. Quest'argomentazione, adattata al caso considerato, può essere schematizzata nel modo seguente.

Invece di considerare soltanto l'atomo radioattivo, si consideri un sistema che comprende anche l'apparato strumentale che accerta la trasformazione radioattiva, per esempio, un contatore Geiger; si supponga, inoltre, che l'apparato strumentale includa un nastro per la registrazione sul quale sia fatto un segno quando scatta il contatore Geiger, e che il nastro sia fatto scorrere a velocità costante, di modo che dalla lettura della posizione del segno sul nastro si possa risalire al tempo in cui è avvenuto il singolo decadimento. Sebbene questo sistema totale sia molto complesso (la sua funzione d'onda dipende dalla sua configurazione microscopica, vale a dire dalle posizioni di tutti i suoi costituenti elementari ), è del tutto legittimo trattare tutto questo sistema dal punto di vista della meccanica quantistica.

Come nel caso del solo atomo che decade, la funzione d'onda del 
sistema totale determina la probabilità della configurazione microscopica ad ogni istante di tempo. Consideriamo questa funzione d'onda ad un tempo molto grande in rapporto al tempo medio di decadimento dell'atomo radioattivo - cioè, ad un tempo in cui siamo praticamente certi che si sia prodotto un segno sul nastro. Ad ogni configurazione microscopica corrisponde una posizione ben definita del segno sul nastro di carta, e la funzione d'onda risulta sparpagliata su tutte queste configurazioni. Vale a dire, questa funzione non assegna un valore numerico ben definito alle configurazioni microscopiche a cui corrisponde una e una sola configurazione macroscopica (il nastro di carta con un segno ben definito da qualche parte), ma assegna valori numerici a una moltitudine di configurazioni (ciascuna delle quali corrisponde al nastro di carta con un segno da qualche parte).

Insomma, la funzione d'onda fornisce soltanto le probabilità di tutte le configurazioni microscopiche possibili, e per le posizioni macroscopiche dà soltanto le probabilità, ma non le posizioni del segno sul nastro di carta. Poiché non rappresenta affatto ciò che osserviamo in laboratorio, cioè un nastro di carta con un segno ben definito da qualche parte, questa funzione non fornisce una descrizione completa degli stati di cose macroscopici. Si ha così — a parte un caveat — una reductio ad absurdum dell'ipotesi di completezza della funzione d'onda. A commento del ragionamento appena esposto, Einstein scrive:

In questo ragionamento, la posizione del segno sul nastro ha la stessa parte che aveva nel ragionamento originario l'istante di disintegrazione. La ragione per cui s'introduce un sistema corredato di meccanismo di registrazione sta in questo. La posizione del segno sul nastro di registrazione è un fatto che rientra interamente nell'ambito dei concetti macroscopici, a differenza dell'istante di disintegrazione di un singolo atomo (ibid.).

\section{Meccanica QuANTistica con L'osservatore}

Il caveat lasciato in sospeso è questo. Se proviamo ad accettare l'interpretazione per cui la descrizione della teoria quantistica dovrebbe essere intesa come una descrizione completa del sistema individuale, siamo costretti a interpretare la posizione del segno sul nastro come qualcosa che non appartiene al sistema in sé, ma la cui esistenza dipende essenzialmente dal fatto che si sia condotta un'osservazione sul 
nastro registratore. Un'interpretazione del genere, certamente, non è affatto assurda da un punto di vista puramente logico; tuttavia, è molto improbabile che ci sia qualcuno disposto a prenderla seriamente in considerazione.

Di certo, come risulta dal passo che abbiamo citato all'inizio di quest'articolo, Feynman provò un senso di disagio di fronte alla possibilità che l'osservatore crea, in qualche modo, la realtà. È difficile credere che un'interpretazione del genere possa essere presa in seria considerazione da molti fisici, perlomeno, per così dire, "nel loro orario di lavoro". La ragione, come ci ricorda Einstein, è chiara: nel campo macroscopico si considera semplicemente certo che ci si debba attenere al programma di una descrizione realistica nello spazio e nel tempo; mentre nel campo microscopico si è più facilmente portati a rinunciare, o almeno a modificare, questo programma.

Per alcuni fisici, invece, il caveat del ragionamento di Einstein è diventato la lezione principale della meccanica quantistica. Il fisico americano John Wheeler, per esempio, è famoso per avere congetturato un "universo partecipativo" in cui le osservazioni fatte nel presente dai fisici sperimentali, o dall'uomo della strada quando osserva il cielo stellato, danno "realtà tangibile" al passato lontano. Si tratta di una concezione in cui le azioni che compiamo adesso possono, in qualche modo, produrre la struttura fisica del passato dell'universo, invece di fornirci, semplicemente, informazioni sul suo passato. È una speculazione ardita che viola il buon senso: come non pensare che i miei occhi abbiano catturato i fotoni emessi da quella stella migliaia d'anni fa perché quei fotoni si stavano già muovendo in quella direzione molto prima che io avessi deciso di andare sul terrazzo e guardare proprio quella stella? Pensare che io abbia contribuito alla formazione della struttura fisica del passato del mondo - influenzando il processo di emissione di quei fotoni che sono finiti nel mio occhio - è ben più che la violazione delle certezze del senso comune a cui la scienza ci ha abituato, sembra piuttosto sconfinare nella megalomania e nella follia.

Se si vogliono evitare le conseguenze stravaganti della seconda opzione, sembrerebbe naturale optare per la prima, cioè assumere un quadro concettuale in cui la funzione d'onda non fornisce una rappresentazione completa del singolo sistema. Le ragioni del perché questa non sia stata la scelta maggioritaria tra i fisici sono molteplici e, tra queste, la convinzione largamente diffusa che siano i fatti sperimentali della meccanica quantistica a rendere impossibile una formulazione comple- 
ta della meccanica quantistica (con buona pace degli sforzi metodologici ed epistemologici di Einstein nel chiarire i termini corretti della questione).

Questa convinzione è stata anche nutrita dai cosiddetti teoremi limitativi di "non esistenza delle variabili nascoste". Si è ritenuto che questi teoremi dimostrino che non è possibile che la casualità quantistica emerga semplicemente dall'ignoranza, in altre parole, che non possano esistere quelle variabili che, nella visione di Einstein, dovrebbero completare la descrizione della realtà fisica fornita dalla funzione d'onda. John Bell, tuttavia, in un suo famoso articolo del 1966 ("On the problem of hidden variables in quantum mechanics", riprodotto in Bell 1987), ha mostrato che questi teoremi limitativi coinvolgono assunzioni immotivate e quindi non giustificano il rigetto del punto di vista di Einstein sull'incompletezza del formalismo quantistico e sull'origine della casualità quantistica. Inoltre, dal 1952, esiste un notevole controesempio all'interpretazione di questi teoremi (ce ne occuperemo diffusamente più avanti).

\section{VARIABILI PRIMITIVe}

Il punto di vista di Einstein può essere riassunto dall'affermazione che oltre alla funzione d'onda devono esistere altre variabili, che chiameremo variabili primitive, in termini delle quali è possibile fornire la descrizione completa di ogni situazione individuale "che si suppone possa esistere indipendentemente da ogni atto di osservazione o di verifica".

Nulla sembrerebbe più lontano dall'interpretazione ortodossa della meccanica quantistica. Sorprendentemente, il punto di vista di Bohr ha alcuni tratti in comune con quello di Einstein.

Per Bohr è in linea di principio impossibile formulare i concetti fondamentali della meccanica quantistica senza usare la meccanica classica. Per dirla con le parole di Landau e Lifshitz, "la meccanica quantistica occupa una posizione assai particolare nell'ambito delle teorie fisiche: essa contiene la meccanica classica come caso limite, e al tempo stesso ha bisogno di questo caso limite per la sua fondazione.” Quindi, la visione ortodossa finisce per fornire al problema della completezza, una risposta che molti cultori dell'ortodossia faticano ancora oggi ad accettare: la funzione d'onda, non fornisce una rappresentazione completa dello stato cose nel mondo; in aggiunta a questa, occorre specifi- 
care i valori di opportune variabili macroscopiche che descrivono ad ogni istante lo stato degli oggetti macroscopici (ad esempio, il segno sul nastro di carta). Nella formulazione di Bohr della meccanica quantistica le variabili macroscopiche giocano un ruolo analogo alla variabili primitive di Einstein.

Secondo Bohr, sono proprio queste variabili a fondare la meccanica quantistica e a rendere il formalismo quantistico coerente ed applicabile allo studio dei fenomeni che osserviamo in laboratorio o in natura. In altre parole, secondo la visione ortodossa, la descrizione completa di uno stato di cose del mondo è dato dalla funzione d'onda e da opportune variabili macroscopiche. Sebbene secondo la visione ortodossa la funzione d'onda non rappresenti niente di reale (celebre è il motto "non esiste un mondo quantistico, esiste solo una descrizione quantistica astratta"), il suo ruolo è quello di governare la statistica delle variabili macroscopiche che rappresentano ciò che è da considerarsi reale, o perlomeno "concreto"; ad esempio, la posizione del segno sul nastro di carta.

\section{IL PROBLEMA DELLA VAGHEZZA}

Si presenta tuttavia un nuovo problema. Nella formulazione originaria dell'interpretazione di Copenaghen, il senso in cui la descrizione completa di uno stato di cose del mondo è dato da dalla funzione d'onda e dalle variabili macroscopiche non è specificato chiaramente. Anche la loro dinamica non è precisata in modo chiaro e non ambiguo: talvolta la dinamica della funzione d'onda è data dall'equazione di Schrödinger e talvolta la dinamica delle variabili macroscopiche è quella fissata dalle leggi della meccanica classica e dell'elettromagnetismo classico; ma quando le variabili classiche interagiscono con le variabili quantistiche, le leggi dinamiche cambiano: la funzione d'onda non evolve più secondo l'equazione di Schrödinger, ma evolve secondo la cosiddetta regola di collasso della funzione d'onda, e le variabili macroscopiche subiscono "salti" casuali, del tutto imprevedibili sulla base delle leggi classiche, salti che sono sono regolati solo statisticamente dalla funzione d'onda.

La difficoltà sollevata da Feynman permane: dov'è la linea di confine tra ciò che è classico e ciò che è quantistico? Quando possiamo trattare un oggetto come classico e quando dobbiamo invece trattarlo come quantistico? In altre parole, la distinzione tra microscopico e 
macroscopico, così come quella tra mondo classico e mondo quantistico, mancano di una definizione precisa e introducono un'ambiguità fondamentale che non può avere alcun posto in una qualunque teoria che abbia la pretesa di precisione fisica. Questa ambiguità e la conseguente difficoltà a comprendere come la dualità tra mondo classico e mondo quantistico possa essere compatibile con il fatto che le mele e le sedie consistano di elettroni e di altre particelle sono alla base della "filosofia della complementarietà" di cui Bohr fu propugnatore; si tratta di una filosofia accondiscendente con le contraddizioni che si compiace dell'utilizzo di concetti ambigui.

\section{MECCANICA QUANTISTICA SENZA L'OSSERVATORE}

C'è un modo molto semplice per uscire dall'ambiguità: anziché introdurre variabili macroscopiche, si introducano variabili microscopiche ben definite a tutte le scale! In questo modo il punto di vista di Bohr si salderebbe con quello di Einstein. All'ovvia domanda del perché la fisica non abbia seguito questa strada, si può dare l'ovvia risposta che costruire teorie è un lavoro che richiede fatica e immaginazione.

La scoperta di nuove teorie, teorie di variabili ben più primitive delle variabili del formalismo quantistico usuale, non è un lavoro così facile quanto interpretare filosoficamente un formalismo già esistente, che è quello che Bohr finisce per fare. La risposta di Bohr è che la ricerca di variabili più primitive della variabili macroscopiche è vana perché non è possibile andare oltre lo schema interpretativo della meccanica quantistica basato sulle variabili macroscopiche. Ma la ragione di questa impossibilità, una volta che si è preso atto dell'inutilità dei teoremi limitativi sulle variabili nascoste, si riduce alla seguente petizione di principio: "perché le nostre capacità cognitive non ci permettono di rappresentare con immagini chiare e non contraddittorie quello che accade nel mondo microscopico".

Comunque la mettiamo, ci troviamo di fronte ad una giustificazione della dottrina di Copenaghen analoga a quella raccontata da Karl Popper (1969): qualcuno potrebbe chiedersi "perché il mare è agitato?", e un altro potrebbe rispondere: "perché Nettuno è molto arrabbiato". Se chiedessimo a costui di portare una prova alla sua spiegazione egli risponderebbe: "non vedi il mare come è agitato? Il mare è sempre agitato quando Nettuno è arrabbiato". 
L'insistenza di Einstein sulla possibilità di arrivare ad una formulazione completa non ambigua e indipendente dall'osservatore sembra l'atteggiamento più naturale se confrontata con le categoriche asserzioni di Bohr riguardanti l'impossibilità, in linea di principio, di una descrizione di questo tipo. Ciò nonostante, la maggioranza dei fisici ritiene che questo dibattito tra Einstein e Bohr, durato decenni, sia stato vinto da Bohr e che questo dibattito sia per lo più filosofico e non suscettibile di alcuna soluzione scientifica.

Tuttavia, oggi possiamo affermare con certezza che quel dibattito è stato risolto, e, in effetti, a favore di Einstein: quello che Einstein desiderava e Bohr riteneva impossibile, in effetti, esiste. Esiste una meccanica quantistica che fornisce una "descrizione completa di ogni situazione reale (individuale) che si suppone possa esistere indipendentemente da ogni atto di osservazione o di verifica", vale a dire, una meccanica quantistica basata su variabili primitive. Anzi, oggi abbiamo diverse teorie di questo tipo. Abbiamo la meccanica bohmiana, anche nota come teoria d'onda pilota di de Broglie-Bohm, che esiste dal 1927, quando fu per la prima volta presentata da Louis de Broglie al congresso Solvay che si tenne in quell'anno, e che fu indipendentemente riscoperta nel 1952, l'anno in cui David Bohm pubblicò due lavori intitolati "A Suggested Interpretation of Quantum Theory in Terms of Hidden Variables", cioè una proposta di interpretazione della teoria quantistica in termini di quelle variabili che sarebbero precluse dai teoremi limitativi a cui abbiamo prima accennato.

In meccanica bohmiana, un sistema di particelle è descritto in parte dalla sua funzione dell'onda che evolve, come di consueto, secondo l'equazione di Schrödinger. Tuttavia, la funzione dell'onda fornisce soltanto una descrizione parziale del sistema. Questa descrizione è completata dalla specificazione delle posizioni reali delle particelle. La loro evoluzione è regolata da una equazione di guida che esprime le velocità delle particelle in termini della funzione d'onda. Perciò in meccanica bohmiana la configurazione di un sistema di particelle evolve secondo un movimento che è in qualche modo governato dalla funzione d'onda. In questa teoria il segno sul nastro di carta è sempre in una posizione definita e il gatto è sempre o vivo o morto, essendo questa sua condizione determinata dalle posizioni delle particelle che lo costituiscono e che hanno sempre, ad ogni istante, valori ben definiti. Le variabili primitive di Einstein sono dunque le posizioni delle particelle e le variabili macroscopiche di Bohr sono funzioni a "grana grossa" delle 
prime che permettono di determinare la manifestazione macroscopica dello stato di cose microscopico. ${ }^{1}$

Nel 1986 i fisici italiani Gian Carlo Ghirardi, Alberto Rimini e Tullio Weber ebbero successo nello sviluppare un'altra teoria di questo tipo, la cosiddetta "teoria GRW". In questa teoria la linea di confine tra mondo classico e mondo quantistico è definita da una nuova costante di natura. Contrariamente a quel che accade nella meccanica quantistica di Bohr, in questa teoria il confine tra mondo classico e mondo quantistico è suscettibile di indagine sperimentale, e sebbene il presente sviluppo tecnologico non permetta ancora di portare a termine esperimenti cruciali, la teoria è in linea di principio falsificabile, perché prevede che su una ben definita scala l'interferenza quantistica sia soppressa.

Secondo la proposta di Ghirardi (2001), le variabili primitive di questa teoria descrivono un campo sullo spazio fisico che può essere identificato, su scala macroscopica, con l'usuale densità di massa degli oggetti fisici. La descrizione che ne deriva, in termini di come la massa degli oggetti si distribuisce nello spazio fisico in accordo con i valori assunti da questo campo, è in completo accordo con la nostra esperienza quotidiana, e include gatti, contatori Geiger e nastri di carta; secondo questa descrizione, il gatto non risulta essere un agglomerato di particelle, ma corrisponde ad una data configurazione del campo che, su scala macroscopica, ne disegna le sembianze familiari.

Quando queste teorie sono analizzate attentamente, emerge una lezione filosofica molto importante: nella loro struttura si possono scorgere caratteristiche molto generali che sono comuni a tutte le "teorie quantistiche senza l'osservatore", ${ }^{2}$ cioè a tutte le formulazioni precise e serie della meccanica quantistica che non sono basate su nozioni vaghe e imprecise come "misura" o "osservatore" (Allori et al., 2008). Meccanica bohmiana e teoria GRW sono teorie che alla fantastica precisione numerica della meccanica quantistica convenzionale fanno corrispondere un'altrettanta precisione concettuale nella formulazione dei loro assiomi. In queste teorie le variabili primitive rappresentano i mattoni da costruzione di tutto ciò che esiste secondo la teoria, dalle parti-

1 Per un approfondimento di questa teoria si veda, ad esempio, D. Dürr et al. (2013) oppure, a livello divulgativo (e in italiano), Allori et al. (2005).

2 La terminologia è di Popper (1967). 
celle subatomiche agli atomi e alle molecole, fino ai tavoli e alle sedie. Ciascuna di queste teorie può essere vista come una realizzazione dell'intuizione di Einstein che la funzione d'onda non fornisce una rappresentazione completa della realtà fisica.

\section{ROBA LOCALE E COMPRENSIBILITÀ}

Un tratto comune delle meccanica bohmiana e della teoria GRW (nella formulazione di Ghirardi) è che le variabili primitive sono variabili locali, cioè variabili che rappresentano "roba" nello spazio che evolve nel corso del tempo o, per dirla in breve, "roba locale". Per designarla, Bell coniò il termine "local beable". ${ }^{3}$ Le robe (o beable) locali

sono le controparti matematiche nella teoria degli eventi reali che accadono in luoghi e a tempi definiti nel mondo reale (e, in quanto tali, distinte dalle tante costruzioni puramente matematiche che si presentano nell? elaborazione delle teorie fisiche, distinte da ciò che può essere reale ma non è localizzato, e distinte dalle 'osservabili' di altre formulazioni della meccanica quantistica, per le quali non abbiamo alcun uso qui) (Bell 1987, p. 205).

È proprio perché è facile produrre fisicamente correlazioni tra la disposizione della roba locale e lo stato di un apparato di misura (o lo stato del nostro cervello), che la roba locale rende intellegibile quello che succede nel mondo. Al contrario, la comprensione delle relazioni

3 Il termine "beable" potrebbe essere tradotto in italiano con "esseribile", ma questo lo carica di una connotazione eccessivamente metafisica e si perde la sottile ironia di Bell, che lo aveva scelto in opposizione al termine "osservabile", che domina la vulgata dei fisici contemporanei. Bell diceva che la sua intenzione non era di spaventare con della 'meta-fisica' coloro che sono dediti alla 'vera-fisica', voleva piuttosto rendere esplicite alcune nozioni che sono già implicite e fondamentali per la meccanica quantistica ordinaria. La sua idea era di trasferire al contesto quantistico la distinzione familiare già nota in teoria classica tra quantità 'fisiche' e "non-fisiche', ad esempio, in elettrodinamica classica si distinguono i campi elettrico e magnetico dai potenziali scalari e vettori, che sono solo una convenienza matematica (ad esempio, non importa che nel gauge di Coulomb il potenziale scalare si propaghi con velocità infinita, perché non si suppone che ci sia davvero). 
tra una teoria senza roba locale e le nostre credenze pre-teoriche $e^{4}$ sarà molto, molto più complessa. In una teoria fisica priva di roba locale dovremmo ricorrere a "leggi-ponte", nel senso dei neo-positivisti, o invocare una qualche versione di funzionalismo, per esempio à la Dennett. Ma potremmo ancora dire che la teoria rende intellegibile quello che succede nel mondo? La domanda è difficile e ci rimanda all'aspetto più delicato e sfuggente del rapporto tra quadro concettuale di una teoria fisica e realtà empirica che non possiamo affrontare qui. Ci limitiamo a poche e scarne osservazioni conclusive.

\section{CONCLUSIONI}

Il quadro concettuale di una teoria fisica è fondato su una struttura matematica che può essere organizzata in modo assiomatico. Gli assiomi riguardano, tra l'altro, la definizione e il comportamento degli enti matematici che svolgono un ruolo essenziale nella formulazione della teoria stessa. Sebbene la corrispondenza tra assiomi e realtà empirica sia sempre molto problematica ed abbia un ineliminabile elemento extra-logico ed intuitivo, essa deve poter essere stabilita, per lo meno pragmaticamente, con un elevato grado di certezza. In altre parole, deve sussistere una qualche relazione tra la struttura matematica della teoria e certi stati di cose, in particolare quelli a cui abbiamo un accesso sensoriale diretto. Come comprese Immanuel Kant, questa relazione è garantita se esiste una "continuità" tra i significati degli enti matematici che intervengono negli assiomi della teoria e concetti dell'esperienza ordinaria (che Kant chiamava "trascendentali"). ${ }^{5}$ Le variabili primitive,

4 Intendiamo le nostre credenze anteriori a qualunque educazione scientifica, credenze in un mondo che contiene oggetti localizzati che hanno certe posizioni nello spazio che cambiano nel corso del tempo.

5 Continuità non significa identità. Mentre per Kant fu facile identificare l'immagine scientifica del mondo con quella manifesta, questo oggi sarebbe impensabile. La fisica moderna ci abituato ad un progressivo allontanamento dallo spazio del senso comune, prima con lo spaziotempo quadridimensionale della teoria della relatività e poi con gli spazi decadimensionali delle teorie di supercorda. Il punto è che quando il mondo della teoria è a "guardato a grana grossa" ne risulta il mondo macroscopico che ci è familiare. "Guardare a grana grossa" ha un significato matematico preciso; significa ricavare la descrizione macroscopica fornita 
proprio perché rappresentano roba locale, svolgono questo ruolo di raccordo tra la teoria e il mondo dell'esperienza ordinaria. È grazie a questa continuità di significato che la teoria fornisce un'immagine del mondo che ci risulta comprensibile. Questo aspetto del rapporto tra quadro concettuale di una teoria fisica e realtà empirica era chiaro ad Einstein che, con acutezza ed estrema semplicità, riassunse così: "una delle grandi scoperte di Immanuel Kant fu il riconoscimento che la costruzione di un mondo esterno reale sarebbe priva di senso senza la sua comprensibilità (Einstein, 1988, p. 530)" .

\section{Bibliografia}

Allori V., Dorato M., Laudisa F., Zanghì N. (2005). La natura delle cose, introduzione ai fondamenti e alla filosofia del la fisica. Roma: Carocci.

Allori V., Goldstein S., Tumula T., Zanghì N. (2008). On the Common Structure of Bohmian Mechanics and the Ghirardi-Rimini-Weber Theory. The British Journal for the Philosophy of Science 59, 353.

Bell J. S. (1987) Speakable and Unspeakable in Quantum Mechanics, Cambridge: Cambridge University Press. Trad. it. Dicibile e indicibile in meccanica quantistica, Adelphi (2010).

Bohm D. (1952) A Suggested Interpretation in Terms of "Hidden Variables": Part I and Part II, Physical Review 85: 166-179 and 180-193.

Dürr, D., Goldstein, S. Zanghì, N. (2013). Quantum Physics Without Quantum

Philosophy. Berlin, Heidelberg: Springer.

Einstein, A. (1988) Fisica e realtà in "Albert Einstein. Opere scelte", a cura di Bellone, E. Torino: Bollati Boringhieri.

Einstein, E. (1979). Autobiografia Scientifica, Torino: Boringhieri.

Einstein, E., Podolsky B., Rosen N. (1935). Can Quantum Mechanical Description of Reality be Considered Complete? , Physical Review 47, 777-780.

Feynman, R. (2002). Feynman Lectures on Gravitation. Cambridge, MA: Perseus.

dalla teoria utilizzando opportune funzioni sullo spazio degli stati microscopici; in questo modo il mondo della nostra esperienza ordinaria è in continuità con il mondo microscopico.

6 È interessante osservare che l'aderenza al Kantismo ortodosso portò Bohr a ritenere che la fisica classica fosse "a priori" e quindi fosse impossibile un'immagine coerente e non contraddittoria del mondo quantistico. Mentre Einstein, andando al cuore dell'insegnamento kantiano, spogliandolo cioè di quegli aspetti che lo sviluppo della fisica aveva reso inadeguati, giunse alla conclusione opposta. 
Ghirardi, G. C. (2001). Un?occhiata alle carte di Dio, Milano: Il Saggiatore.

Ghirardi G.C., Rimini A., Weber T. (1986). Unified Dynamics for Microscopic and Macroscopic Systems, Physical Review D 34, 470.

Landau, L., Lifsits, E. (2010) Fisica Teorica 3. Meccanica quantistica. Teoria non relativistica. Editori Riuniti Univ. Press.

Popper, K.R. (1967). Quantum Mechanics without “The Observer”. In: Bunge M. (eds) Quantum Theory and Reality. Studies in the Foundations, Methodology and Philosophy of Science, 7-44. Berlin, Heidelberg: Springer.

Popper, K.R. (1969). Scienza e filosofia, Torino: Einaudi.

Schrödinger., E. (1935). Die Gegenvärtige Situation in der Quantenmechanik, Naturwissenschaften 23, 807-812; Trad. ingl. di J. D. Trimmer: The Present Situation in Quantum Mechanics: a Traslation of Schrödinger's "Cat Paradox" Paper, in: Wheeler, W. H. Zurek, Quantum Theory and Measurement, Princeton University Press, Princeton (1983). 